
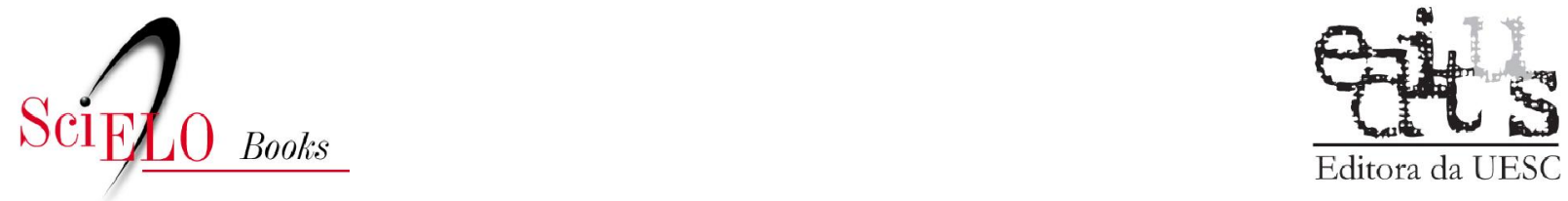

Editora da UESC

\title{
3 - Estado da arte sobre a taxonomia e filogenia de Ectatomminae
}

\author{
Gabriela P. Camacho \\ Rodrigo M. Feitosa
}

\section{SciELO Books / SciELO Livros / SciELO Libros}

CAMACHO, GP., and FEITOSA, RM. Estado da arte sobre a taxonomia e filogenia de Ectatomminae. In: DELABIE, JHC., et al., orgs. As formigas poneromorfas do Brasil [online]. Ilhéus, BA: Editus, 2015, pp. 23-32. ISBN 978-85-7455-441-9. Available from SciELO Books $<\underline{\text { http://books.scielo.org }>\text {. }}$

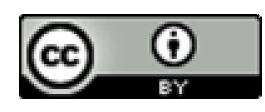

All the contents of this work, except where otherwise noted, is licensed under a Creative Commons Attribution $\underline{4.0 \text { International license. }}$

Todo o conteúdo deste trabalho, exceto quando houver ressalva, é publicado sob a licença Creative Commons Atribição 4.0.

Todo el contenido de esta obra, excepto donde se indique lo contrario, está bajo licencia de la licencia $\underline{\text { Creative }}$ Commons Reconocimento 4.0. 


\title{
Estado da arte sobre a taxonomia e filogenia de Ectatomminae
}

\author{
Gabriela P. Camacho, Rodrigo M. Feitosa
}

\section{Resumo}

Ectatomminae é formada por quatro gêneros: Ectatomma Fr. Smith e Typhlomyrmex Mayr, exclusivos da região Neotropical; Rhytidoponera Mayr, que ocorre apenas na Região Australiana; e Gnamptogenys Roger, presente nas regiões Neotropical, Neártica, Indo-malaia e Australiana. Em termos de diversidade, a subfamília é composta por 266 espécies no mundo todo, com 112 ocorrendo na Região Neotropical, e 50 presentes no Brasil. Pouco se sabe sobre as relações filogenéticas internas em Ectatomminae, mas muitos estudos têm sido feitos na tentativa de se compreender a taxonomia e a biologia dos gêneros, especialmente daqueles que ocorrem no Brasil. Atualmente, a identificação das espécies de Ectatomminae é relativamente fácil, contando com chaves dicotômicas eficientes para os três gêneros. Quanto à sua biologia, a subfamília apresenta espécies nidificando no solo, na serapilheira, em troncos em decomposição ou mesmo no estrato arbóreo e arbustivo de florestas, incluindo o dossel. São na maioria predadoras generalistas, com algumas espécies que coletam honeydew de hemípteros e néctar-extrafloral de plantas. Há ainda o registro de pelo menos um caso de parasitismo social nesta subfamília.

CAMACHO, Gabriela P.; FEITOSA, Rodrigo M. Estado da arte sobre a taxonomia e filogenia de Ectatomminae. In: DELABIE, Jacques H. C. et al. As formigas poneromorfas do Brasil. Ilhéus: Editus, 2015. p. 23-32. 


\section{Abstract}

State of the art of the taxonomy and phylogeny of the subfamily Ectatomminae Ectatomminae consists of four genera: Ectatomma Fr. Smith and Typhlomyrmex Mayr, exclusive from the Neotropical region; Rhytidoponera Mayr, which occurs only in the Australian region; and Gnamptogenys Roger, present in Neotropical, Nearctic, Indo-Malayan and Australian regions. In terms of diversity, the subfamily is composed of 266 species worldwide, with 112 representatives occurring in the Neotropics, 50 of them in Brazil. Little is known about the phylogenetic relationships within the Ectatomminae, but many studies have been conducted in an attempt to understand the taxonomy and biology of the ectatommine genera, especially in Brazil. Currently, the identification of Ectatomminae species is a relatively easy task, with efficient dichotomous keys available for the three genera in Brazil. In regards to their biology, the subfamily has species nesting in soil, leaf litter, rotten logs and even in the tree and shrub layer of forests, including the canopy. The species are usually generalist predators, with some collecting honeydew from Hemiptera and extrafloral nectar from plants. In addition, there is at least one record of social parasitism in ectatommines.

\section{Sistemática de Ectatomminae}

A subfamília Ectatomminae abriga formigas pequenas a médias, monomórficas e amplamente distribuídas, sendo formada por quatro gêneros: Ectatomma Fr. Smith e Typhlomyrmex Mayr, exclusivos da Região Neotropical; Rhytidoponera Mayr, que ocorre apenas na Região Australiana; e Gnamptogenys Roger, presente nas regiões Neotropical, Neártica, Indo-malaia e Australiana. Apesar de sua abundância, ainda há muito a se conhecer sobre as relações filogenéticas entre estes gêneros.

Os gêneros ectatommíneos foram primeiramente posicionados na subfamília Ponerinae, mais precisamente na tribo Ectatommini (com exceção de Typhlomyrmex), por Brown (1954) e por vários autores subsequentemente (BOLTON, 1994; 1995). A primeira revisão para a tribo classificou como pertencentes à Ectatommini Acanthoponera, Aulacopone, Discothyrea, Ectatomma, Gnamptogenys, Heteroponera, Paraponera, Proceratium e Rhytidoponera (BROWN, 1958). Neste trabalho, através de análises morfológicas, Brown estabelece uma maior relação entre Gnamptogenys e Rhytidoponera e os gêneros Acanthoponera e Heteroponera, e propõe Ectatomma como uma linhagem basal dentro da tribo, juntamente com Paraponera. O gênero Typhlomyrmex, até então, era considerado como pertencente à subtribo Typhlomyrmecini, posteriormente elevada à tribo (BROWN, 1965).

Utilizando-se de caracteres morfológicos, Lattke (1994) apresenta a primeira análise filogenética para a tribo, demonstrando a polifilia de Ectatommini. De acordo com a nova classificação, Ectatommini passou a ser formada por dois grupos: o clado Heteroponera, formado por Acanthoponera e Heteroponera, e o clado Ectatomma, com Gnamptogenys, Ectatomma e Rhytidoponera (Figura 3.1A). Mais tarde, através da ampliação da matriz de caracteres e da inclusão de novos táxons, Keller (2000) desenvolveu uma nova análise filogenética para o grupo (Figura 3.1B). Seus resultados confirmaram a polifilia da tribo, apontando a monofilia dos grupos Heteroponera e Ectatomma, com Typhlomyrmex apresentando uma grande distância filogenética destes grupos.

Dadas tais instabilidades nas classificações de Ectatommini e demais tribos de Ponerinae, Bolton (2003) desenvolveu um estudo morfológico abrangente e a dividiu em seis subfamílias, sendo uma delas a nova subfamília Ectatomminae, composta pelos gêneros Ectatomma, Gnamptogenys, Rhytidoponera e Typhlomyrmex. Os demais gêneros que compunham a tribo Ectatommini foram classificados nas subfamílias Heteroponerinae (Acanthoponera, Aulacopone e Heteroponera) e Proceratiinae (Discothyrea, Probolomyrmex e Proceratium). As subfamílias originadas do trabalho de Bolton (2003) foram por muito tempo chamadas de poneromorfas, devido a inferências sobre as relações entre elas com base na morfologia externa.

Apenas recentemente, com a ampliação das filogenias utilizando dados moleculares, as relações entre as subfamílias até então consideradas 
Figura 3.1 - Relações entre os gêneros de Ectatommini com as propostas para a reclassificação da tribo segundo (A) Lattke (1994) e (B) Keller (2000). As barras azuis mostram a posição dos gêneros ectatommíneos incluídos nas análises (Modificado de FEITOSA, 2011).
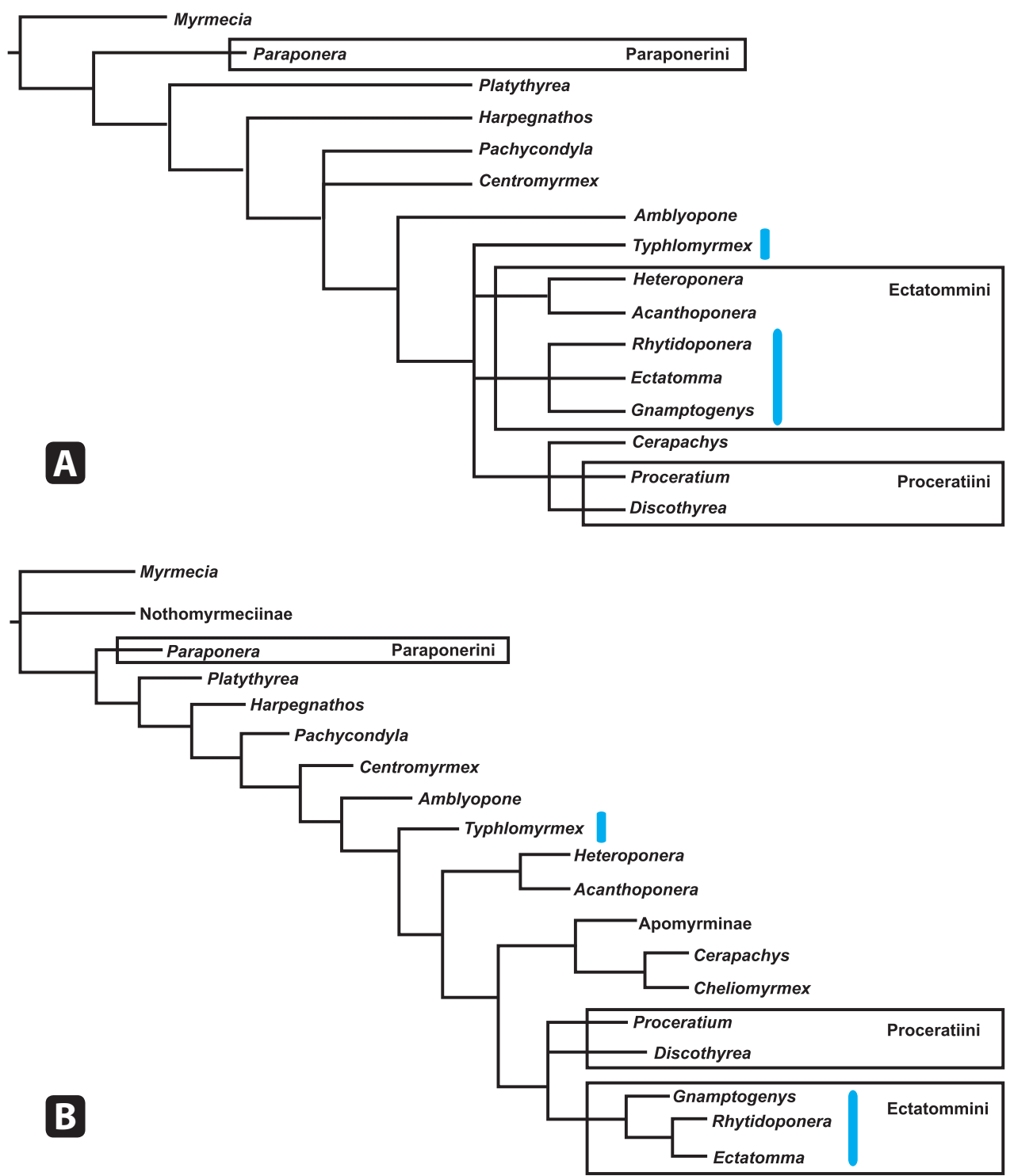

poneromorfas foram devidamente estudadas. Diversos trabalhos demonstraram que, na verdade, as subfamílias Ectatomminae e Heteroponerinae formam juntas o complexo ectaheteromorfo e, ao lado de Formicinae e Myrmicinae, compõem o atual clado formicoide (Brady et al., 2006; Moreau et al., 2006; Ouellette et al., 2006; Rabeling et al., 2008) (Figura 3.2). Em adição Keller (2011), utilizando exclusivamente caracteres morfológicos, comprova a parafilia do complexo poneromorfo e a relação de grupo-irmão entre Ectatomminae e Heteroponerinae (grupo ectaheteromorfo).

A taxonomia global e as relações filogenéticas internas em Ectatomminae ainda não foram estudadas profundamente, embora revisões taxonômicas dos gêneros que a compõem estejam sendo conduzidas. O gênero Gnamptogenys é, certamente, o mais estudado de Ectatomminae. A primeira revisão do gênero foi feita por Brown (1958), com estudos posteriores conduzidos por Brandão e Lattke (1990) e Lattke (1992), com ênfase no grupo minuta. Além disso, Lattke (1995) apresenta uma revisão para as espécies neotropicais e Lattke (2004), para as espécies do Velho Mundo. Além destes trabalhos, Lattke (1990), Fernández (1991), Fernández et al., (1996) e Lattke et al. (2008) estudaram as espécies que ocorrem na Venezuela e na Colômbia, Wu; Wang (1995), Xu; Zhang (1996), Zhou (2001) forneceram chaves para as espécies da China e Shattuck (2000) fez a sinopse das espécies australianas. Desde então, espécies novas encontradas para o grupo têm sido descritas (LATTKE, 2002; LATTKE et al., 
Figura 3.2 - Sumário das relações filogenéticas entre subfamílias de formigas reconstruídas com base em dados moleculares. Os triângulos representam a diversidade de cada subfamília (Modificado de Keller, 2011).

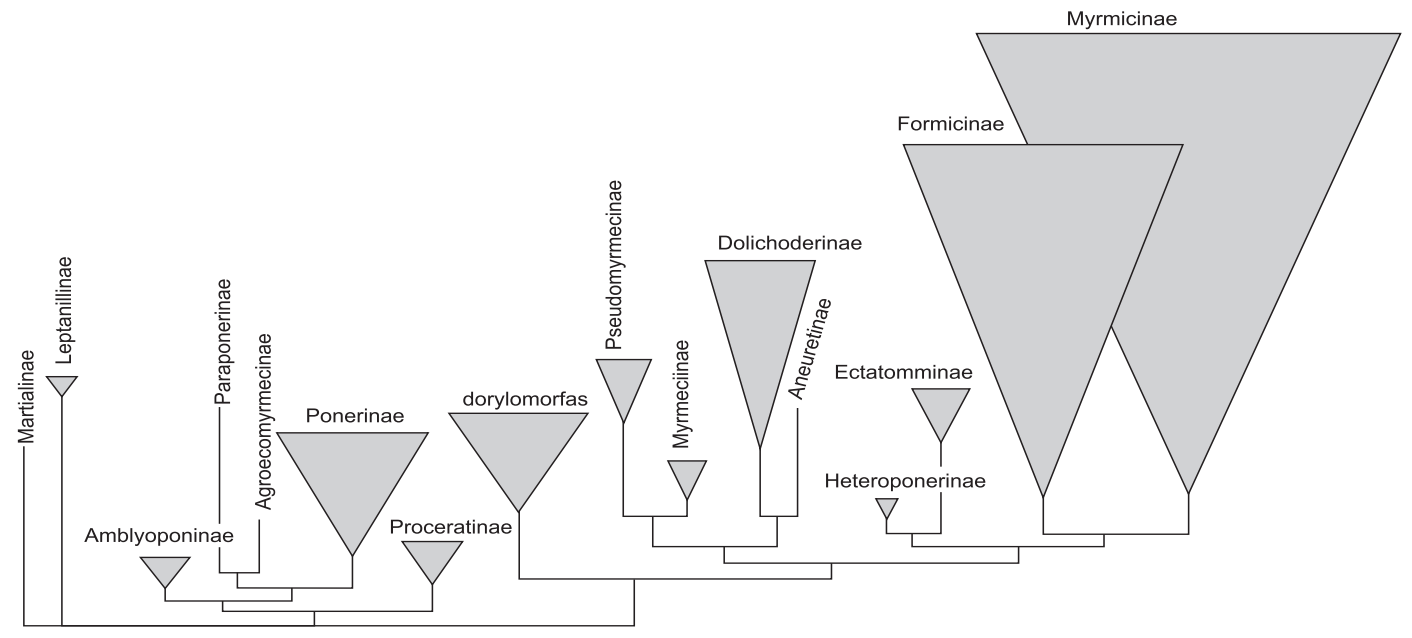

2004; LATTKE et al., 2007; PACHECO et al., 2004), mas sabe-se que muitas ainda estão por descrever. Mais recentemente, Camacho (2013) apresentou uma revisão taxonômica para as espécies do grupo striatula que ocorrem no Brasil, redescrevendo seis espécies e descrevendo quatro espécies novas.

Quanto aos demais gêneros, poucos estudos foram conduzidos nos últimos anos. Desde a revisão de Ectatomma por Brown (1958), chaves de identificação para a maior parte das espécies foram oferecidas por Kugler; Brown (1982), Fernández (1992) (para as espécies da Colômbia) e Arias-Penna (2008). O gênero Rhytidoponera foi revisado por Brown (1958). Desde então, foram produzidas chaves de identificação para as espécies do grupo impressa na Austrália e Nova Guiné e para as espécies da Nova Caledônia, Austrália e Nova Zelândia (HETERICK, 2009; TAYLOR, 1987; WARD, 1980; 1984), além da sinopse taxonômica para as espécies australianas (SHATTUCK, 2000). Por fim, o gênero Typhlomyrmex foi revisado por Brown (1965), que fornece ainda uma chave de identificação para as espécies conhecidas até então. Mais recentemente, uma nova espécie foi descrita (Lacau et al., 2004) e uma chave para as espécies de Typhlomyrmex da Colômbia foi produzida (Lacau et al., 2008).

\section{Representantes de Ectatomminae no Brasil}

\section{Ectatomma Smith, 1858}

Ectatomma pode ser considerado o gênero mais comumente coletado da subfamília na Região Neotropical, sendo endêmico desta região (Kugler; Brown, 1982), com 15 espécies conhecidas até o momento. No Brasil, são conhecidas 11 espécies, caracterizadas por serem formigas grandes, abundantes e conspícuas e com uma clara preferência por hábitats quentes.

As espécies do gênero são caracterizadas pela presença de tubérculos no dorso do pronoto, pelo mesonoto e propódeo formando duas convexidades distintas em vista lateral e pelo espiráculo propodeal alongado, elíptico ou em forma de fenda, porém nunca arredondado (ARIAS-PENNA, 2008) (Figura 3.3). A identificação das espécies de Ectatomma é relativamente simples, baseada principalmente no número, forma e posição dos tubérculos pronotais e esculturação do corpo e conta com uma chave amigável (ARIAS-PENNA, 2008).

Ectatomma tem hábitos generalistas e oportunistas (BROWN, 1958). São predadoras generalistas de diversos artrópodes e anelídeos, além de coletarem secreções açucaradas de hemípteros e néctar extrafloral de algumas plantas (ARIAS -PENNA, 2008). Algumas espécies de Ectatomma também tem sido consideradas importantes predadoras de insetos pragas em diversos agroecossistemas (PÉREZ-LACHAUD et al., 2006). O forrageio é solitário em sua maioria, mas pode também se dar em pares ou em grupo. Também é conhecido o comportamento de cleptobiose intraespecífica para o gênero, em que operárias especializadas adentram colônias vizinhas e roubam alimentos coletados por elas, como resposta à limitação de recursos (GUENARD; MCGLYNN, 2013). Também como resposta à escassez de alimentos, pode haver predação de outras espécies de formigas (LIMA; ANTONIALLI-JÚNIOR, 2013). 
Figura 3.3 - Ectatomma tuberculatum, operária em (a) vista frontal e (b) vista lateral. Foto: April Nobile (Antweb, CASENT0173380).

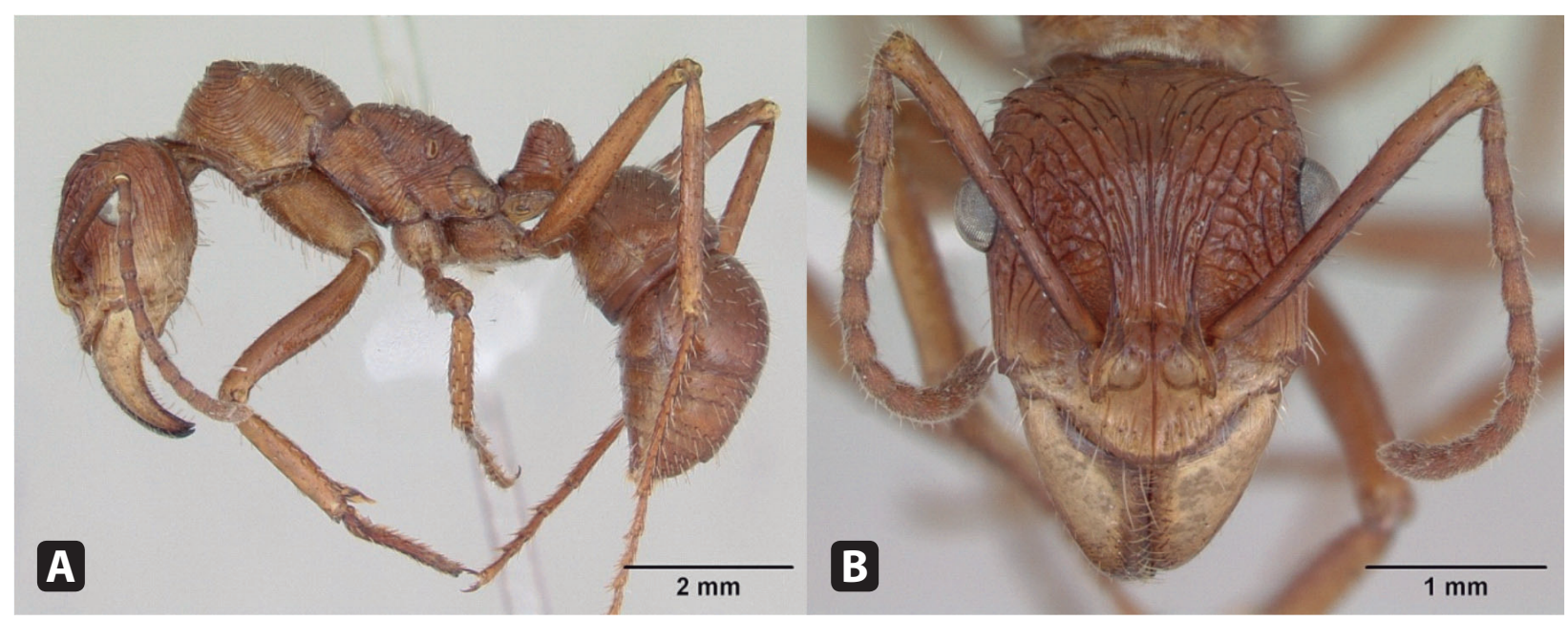

Os ninhos, em geral, são simples, construídos sob o solo e em alguns casos em plantas, inclusive em ambientes urbanos. Em algumas espécies, os ninhos podem alcançar até 3,6 m de profundidade e apresentar de três a dez câmaras (Vieira et al., 2007). As operárias podem apresentar divisão de tarefas ao longo da vida, com operárias jovens realizando tarefas internas à colônia e as mais velhas desempenhando papéis externos (Vieira et al., 2010). As colônias podem ser mono ou poligínicas, apresentando também poliginia facultativa, microginia e parasitismo social (FEITOSA et al., 2008; HORA et al. 2005; LENOIR et al., 2011).

\section{Gnamptogenys Roger, 1863}

Gnamptogenys é o único gênero de Ectatomminae que não está restrito a uma única região biogeográfica, ocorrendo na Região Neotropical e Neártica, do sul dos Estados Unidos ao centro da Argentina, além das regiões Indo-malaia e Australiana, do sul e sudeste da Ásia à Oceania. A maior diversidade do gênero, no entanto, encontra-se nos Neotrópicos, onde 90 espécies são reconhecidas até o momento, mais da metade do total. O gênero Gnamptogenys compreende 140 espécies descritas para o mundo todo e, no Brasil, 33 espécies são conhecidas até o momento.

Segundo Lattke (1995), as características que podem ser consideradas sinapomórficas para o gênero são a presença de uma seta única, ereta e móvel no ápice da protíbia e um espinho ou tubérculo no dorso da metacoxa (Figura 3.4), além da maioria das espécies apresentar o corpo coberto por cóstulas regulares e paralelas. No entanto, as duas últimas características estão ausentes em algumas espécies. Os caracteres geralmente usados para diagnosticar o gênero são relativamente complexos dadas as convergências e perdas secundárias recorrentes no grupo (LATTKE, 1995). Além dos caracteres acima mencionados, é possível identificar o gênero pelas antenas com funículos filiformes, nunca formando uma clava antenal; borda anterior do clípeo com uma lamela estreita de tamanho variável; borda anterior do mesepisterno formando uma lamela estreita; pronoto sempre desarmado, sem espinhos ou tubérculos; espiráculo propodeal arredondado, nunca em forma de fenda; mesonoto nunca convexo e proeminente. As espécies que compõem Gnamptogenys podem ser facilmente diferenciadas das espécies do gênero próximo Ectatomma, por apresentarem o pronoto e mesonoto sem espinhos ou tubérculos (armados em Ectatomma) e pelo mesonoto não proeminente em perfil formando, juntamente com o propódeo, uma superfície contínua ou semicontínua.

Pouco se sabe sobre a biologia das espécies que compõem o gênero, mas a maioria é habitante de florestas úmidas, onde nificam em madeira decomposta, na serapilheira e, por vezes, embaixo de pedras, no solo e, raramente, na vegetação. A maioria das espécies é predadora generalista, porém há espécies especializadas em certos diplópodos (grupo rastrata), coleópteros e outras formigas (G. hartmanni e G. horni) (LATTKE, 1995). O forrageamento é feito ao nível do solo, embaixo da serapilheira, abaixo do solo e na vegetação herbácea. Operárias forrageiam solitariamente, porém pode haver recrutamento no caso de presas grandes. Os ninhos maduros são pequenos, raramente excedendo 500 adultos (LATTKE, 1995). 
Figura 3.4 - Gnamptogenys striatula, operária em (a) vista frontal e (b) vista lateral. Foto: April Nobile (Antweb, JTLC000010271).

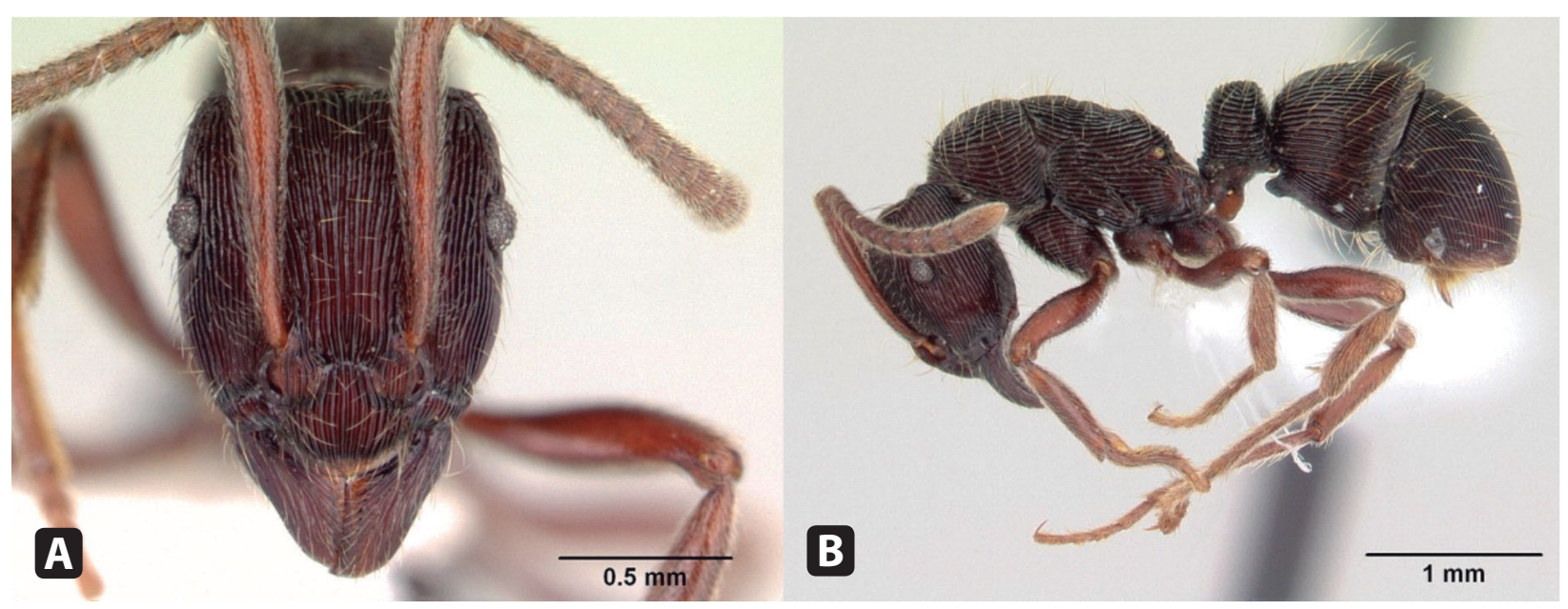

A reprodução ocorre no princípio da estação das chuvas, como na maioria das espécies de formigas neotropicais. Algumas espécies são monogínicas, podendo haver poliginia e até reprodução por gamergates em alguns casos. A reprodução pode ocorrer pela produção de gines ou pela fissão de ninhos maduros, de acordo com a espécie (LATTKE, 1990). O gênero é amplamente coletado dado à sua abundância e diversidade, sendo apontado como um importante indicador de qualidade do hábitat (Pacheco et al., 2013). Sua aparentemente longa história evolutiva, sua diversidade de sistemas de reprodução e sua ampla dieta torna o gênero alvo de vários estudos (BLATRIX; JAISSON, 2000; COGNI; OLIVEIRA, 2004; FOWLER, 1993).

A identificação das espécies do gênero Gnamptogenys no Brasil tem como principal referência o trabalho de Lattke et al. (2007), que apresenta uma chave para as espécies neotropicais. A identificação é baseada principalmente no padrão de esculturação e formato da cabeça, mesossoma e pecíolo. Recentemente, os grupos striatula e rastrata foram revisados para o Brasil. Nestes trabalhos, as espécies conhecidas foram redescritas e seis novas espécies foram descritas (CAMACHO, 2013; GUALBERTO, 2013).

\section{Typhlomyrmex Mayr, 1862}

Typhlomyrmex são formigas endêmicas da Região Neotropical, sendo encontradas do México ao noroeste da Argentina. Das sete espécies conhecidas, seis ocorrem no Brasil: T. clavicornis Emery; T. foreli Santschi; T. major Santschi; T. meire Lacau, Villemant e Delabie; T. pusillus Emery e T. rogenhoferi Mayr. As características diagnósticas para o gênero são os olhos compostos atrofiados e o pecíolo pedunculado e amplamente inserido na face anterior do gáster (LATTKE, 2003) (Figura 3.5).

A identificação das espécies deste gênero pode ser feita através do trabalho de Lacau et al., (2008), que apresenta uma chave para as espécies que ocorrem na Colômbia. A chave compreende cinco espécies, das quais três já são conhecidas (T. major, T. pusillus e T. rogenhoferi) e duas são morfotipos que potencialmente representam espécies novas para a ciência, ainda não formalmente descritas.

O gênero Typhlomyrmex é composto por espécies de tamanho pequeno, crípticas e predadoras, que nidificam em troncos em decomposição ou no solo de florestas úmidas (LATTKE, 2003). Nada ainda se conhece sobre seus hábitos de forrageamento e dieta.

\section{Perspectivas}

Apesar de relativamente comuns e conspícuos, os gêneros que compõem a subfamília Ectatomminae ainda carecem de estudos. Apesar de Ectatomma ser um gênero de taxonomia amigável, pouco se sabe sobre as relações evolutivas entre as espécies que o compõem. No momento, pesquisadores colombianos e franceses estão trabalhando em colaboração para elucidar tais relações e rever os limites entre as espécies conhecidas (POTEAUX et al., dados não publicados; POTEAUX et al., este volume).

Mesmo sendo um gênero composto por apenas sete espécies, o gênero Typhlomyrmex é o 
Figura 3.5 - Typhlomyrmex rogenhoferi, operária em (a) vista frontal e (b) vista lateral. Foto: Fotógrafo desconhecido (Antweb, CASENT0004700).

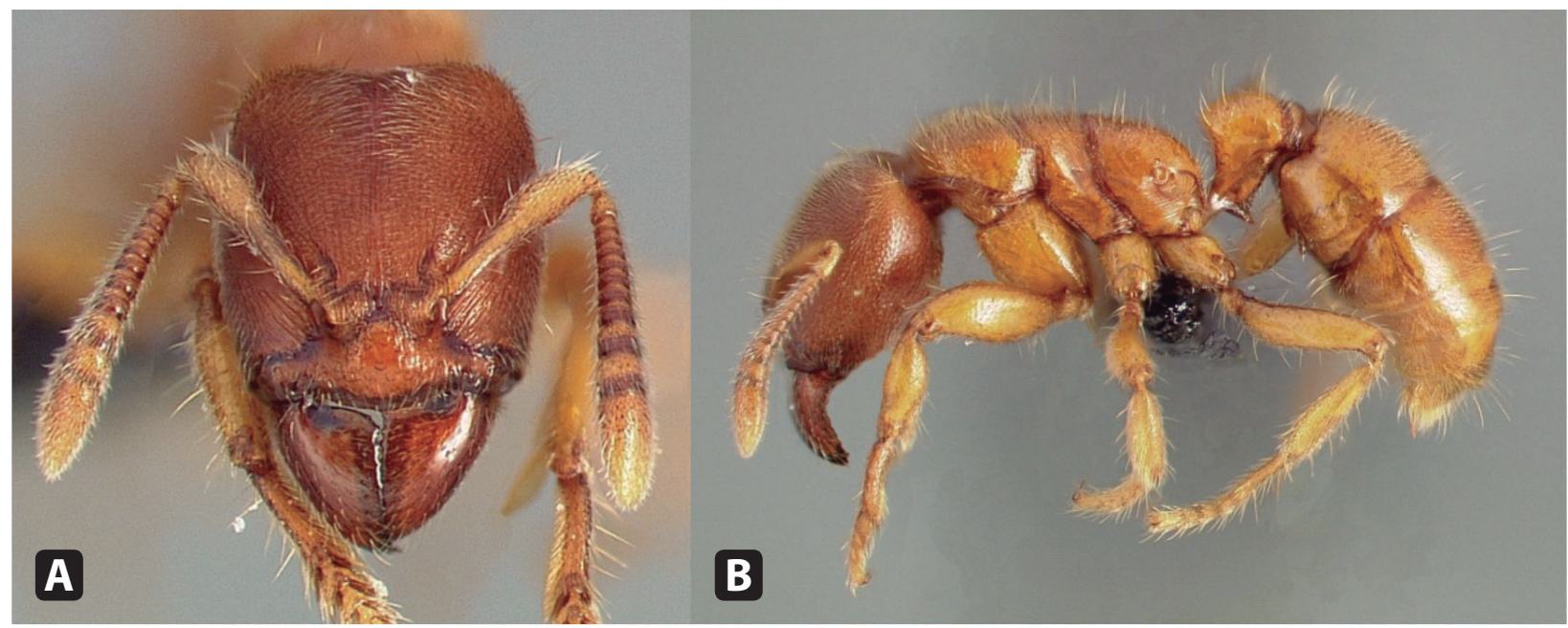

que mais carece de estudos, tanto a respeito de sua taxonomia, quanto da sua biologia e evolução. Nos últimos anos, uma nova espécie foi descrita (LACAU et al., 2004), contribuindo para a taxonomia do grupo, mas trabalhos mais amplos, de revisão e filogenia, devem ser considerados.

O gênero Gnamptogenys, por sua vez, também não conta com nenhum estudo que abranja as relações entre as espécies e a irradiação que ocorreu no grupo. No entanto, esforços consideráveis têm sido feitos nos últimos anos no estudo do gênero. Duas dissertações de mestrado (CAMACHO, 2013; GUALBERTO, 2013) revisaram taxonomicamente os grupos striatula e rastrata, respectivamente, oferecendo redescrições para onze espécies e descrevendo seis novas espécies para o Brasil. Além disso, está em desenvolvimento um trabalho dos autores deste capítulo que visa entender as relações evolutivas do grupo striatula de Gnamptogenys em toda a Região Neotropical, através do uso de ferramentas morfológicas e, pela primeira vez, moleculares. No entanto, devido ao grande número de espécies e à sua distribuição disjunta, muito ainda resta ser estudado em relação ao gênero.

Recentemente, Feitosa (2011) apresenta um estudo extremamente amplo sobre Heteroponerinae, abrangendo análises filogenéticas morfológicas. $\mathrm{O}$ autor evidencia os limites morfológicos frágeis que separam Ectatomminae e Heteroponerinae e sugere a sinonímia de Heteroponerinae, o que agregaria mais três gêneros à Ectatomminae. A publicação deste estudo em breve terá forte impacto sobre a taxonomia de Ectatomminae.

\section{Referências}

ARIAS-PENNA, T. M. Subfamilia Ectatomminae. In: JIMÉNEZ, E; FERNÁNDEZ, F; ARIAS, T.M; LOZANO-ZAMBRANO, F.H. (org). Sistemática, Biogeografía y Conservación de las Hormigas Cazadoras de Colombia. Bogotá D.C., Colombia, Instituto de Investigación de Recursos Biológicos Alexander von Humboldt, p 53-107, 2008.

BLATRIX, R.; JAISSON, P. Optional gamergates in the queenright ponerine ant Gnamptogenys striatula Mayr. Insectes Sociaux, v. 47, p. 193-197, 2000.

BOLTON, B. Identification Guide to the Ant Genera of the World. Harvard University Press, Cambridge, Mass. 1994.

BOLTON, B. A New General Catalogue of the Ants of the World. Harvard University Press, Cambridge, Mass. 1995.

BOLTON, B. Synopsis and classification of Formicidae. Memoirs of the American Entomological Institute, v. 71, p. 1-370, 2003.

BRADY, S. G.; SCHULTZ, T. R.; FISHER, B. L; WARD, P. S. Evaluating alternative hypotheses for the early evolution and diversification of ants. Proceedings of the National Academy of Sciences of the United States of America, v. 103, p. 18172-7, 2006.

BRANDÃO, C. R. F.; LATTKE, J. E. Description of a new Ecuadorean Gnamptogenys species (Hymenoptera: Formicidae), with a discussion on the status of the Alfaria group. Journal of the New York Entomological Society, v. 98, p. 489-494, 1990. 
BROWN, W. L., JR. Remarks on the internal phylogeny and subfamily classification of the family Formicidae.

Insectes Sociaux, v. 1, p. 21-31, 1954.

BROWN, W. L., JR. Contributions toward a reclassification of the Formicidae. II. Tribe Ectatommini (Hymenoptera). Bulletin of the Museum of Comparative Zoology, v. 118, p. 173-362, 1958.

BROWN, W. L., JR. Contributions to a reclassification of the Formicidae. IV. Tribe Typhlomyrmecini (Hymenoptera). Psyche. Cambridge, v. 72, p. 65-78, 1965.

CAMACHO, G. P. Estudo taxonômico do grupo striatula de Gnamptogenys Roger (Hymenoptera, Formicidae, Ectatomminae) para o Brasil.

Dissertação de mestrado. Universidade Federal de Viçosa, Viçosa, 2013.

COGNI, R.; OLIVEIRA, P. S. Patterns in foraging and nesting ecology in the neotropical ant Gnamptogenys moelleri (Formicidae, Ponerinae). Insectes Sociaux, v. 51, p. 123-30, 2004.

FEITOSA, R. M.; HORA, R. R.; DELABIE, J. H. C.; VALENZUELA, J.; FRESNEAU, D. A new social parasite in the ant genus Ectatomma F. Smith (Hymenoptera, Formicidae, Ectatomminae). Zootaxa, v. 1713, p. 47-52, 2008.

FEITOSA, R.S.M. Revisão Taxonômica e Análise Filogenética de Heteroponerinae (Hymenoptera, Formicidae). Tese de Doutorado. Universidade de São Paulo, São Paulo, 2011.

\section{FERNÁNDEZ, C. F. Hormigas Cazadoras}

(Hymenoptera: Formicidae: Ponerinae) de Colombia. Trabajo de grado de Biología, Departamento de Biología, Facultad de Ciencias, Universidad Nacional de Colombia, Bogota, Colombia, 1990. p. 355.

FERNÁNDEZ, F. Las hormigas cazadoras del género Ectatomma (Formicidae: Ponerinae) en Colombia. Caldasia, v. 16, p. 551-64.

FERNÁNDEZ, F.; PALACIO, E. E.; MACKAY, W. P.; MACKAY, E. S. Introduccion al estudio de las hormigas (Hymenoptera: Formicidae) de Colombia. In: ANDRADE, M.; GARCIA, G.; FERNÁNDEZ, F.(org). Insectos de Colombia, Estudios Escogidos. Academia Colombiana de Ciencias Exactas Físicas y Naturales; Universidad Javeriana, Santafé de Bogotá, 1996. p. 349-412.

FOWLER, H. G. Use of fallen cocoa pods by ants (Hymenoptera: Formicidae) in southeastern Brazil. Journal of the Kansas Entomological Society, v. 66, p. 444-6, 1993.
GUALBERTO, M.P. Estudo Taxonômico do Complexo Rastrata, Gênero Gnamptogenys Roger, 1863 (Hymenoptera: Formicidae: Ectatomminae) no Brasil. Dissertação de mestrado. Instituto Nacional de Pesquisas da Amazônia, Manaus, 2013.

GUENARD, B; MCGLYNN, T.P. Intraspecific Thievery in the Ant Ectatomma ruidum is Mediated by Food Availability. Biotropica, v. 45, n. 4, p. 497-502, 2013.

HETERICK, B. E. A guide to the ants of southwestern Australia. Records of the Western Australian Museum, v. 76, p. 1-206, 2009.

HORA, R. R.; DOUMS, C.; POTEAUX, C.; FÉNÉRON, R.; VALENZUELA, J.; HEINZE, J.; FRESNEAU, D. Small queens in the ant Ectatomma tuberculatum: a new case of social parasitism. Behavioral Ecology and Sociobiology, v. 59, p. 285-92, 2005.

KELLER, R. A. Cladistics of the tribe Ectatommini (Hymenoptera: Formicidae): A reappraisal. Insect Systematics; Evolution, v. 31, p. 59-69, 2000.

KELLER, R. A. A phylogenetic analysis of ant morphology (Hymenoptera: Formicidae) with special reference to the poneromorph subfamilies. Bulletin of the American Museum of Natural History, v. 355, p. 1-90, 2011.

KUGLER, C.; BROWN, W. L., JR. Revisionary and other studies on the ant genus Ectatomma, including the description of two new species. Search Agriculture. Ithaca, N. Y., v. 24, p. 1-8, 1982.

LACAU, S.; VILLEMANT, C.; DELABIE, J. H. C. Typhlomyrmex meire, a remarkable new species endemic to Southern Bahia, Brazil (Formicidae: Ectatomminae). Zootaxa, v. 678, p. 1-23, 2004.

LACAU, S.; VILLEMANT, C.; JAHYNY, B.; DELABIE, J. H. C. Typhlomyrmex Mayr, 1862: un genre meconnu de petites fourmis cryptiques et predatrices (Ectatomminae: Typhlomyrmecini). In: JIMÉNEZ, E.; FERNÁNDEZ, F.; ARIAS, T.M.; LOZANOZAMBRANO, F.H. (org). Sistemática, Biogeografía y Conservación de las Hormigas cazadoras de. Colombia. Instituto de Investigación de Recursos Biológicos Alexander von Humboldt, Bogotá D.C., Colombia, 2008, p. 239-83.

LATTKE, J.; FERNANDEZ, F.; ARIAS, T.; PALACIO; MACKAY, W. P.; MACKAY, E. Género Gnamptogenys. In: JIMÉNEZ, E.; FERNÁNDEZ, F.; ARIAS, T.M.; LOZANO-ZAMBRANO, F.H. (org). Sistemática, Biogeografía y Conservación de las Hormigas Cazadoras de Colombia. Instituto de Investigación de Recursos Biológicos Alexander von Humboldt, Bogotá D.C., Colombia, 2008, p. 66-100. 
LATTKE, J. E. Revisión del género Gnamptogenys Roger en Venezuela (Hymenoptera: Formicidae). Acta Terramaris, v. 2, p. 1-47, 1990.

LATTKE, J. E. Revision of the minuta-group of the genus Gnamptogenys (Hymenoptera, Formicidae). Deutsche Entomologische Zeitschrift, v. 39, p. 123-9, 1992.

LATTKE, J. E. Phylogenetic relationships and classification of ectatommine ants (Hymenoptera: Formicidae). Entomologica Scandinavica, v. 25, p. 105-19, 1994.

LATTKE, J. E. Revision of the ant genus Gnamptogenys in the New World (Hymenoptera: Formicidae). Journal of Hymenoptera Research, v. 4, p. 137-93, 1995.

LATTKE, J. E. Nuevas especies de Gnamptogenys Roger, 1863 de América (Hymenoptera: Formicidae: Ponerinae). Entomotropica, v. 17, p. 135-44, 2002.

LATTKE, J. E. Subfamilia Ponerinae. In: FERNÁNDEZ, F. Introducción a las Hormigas de la Región Neotropical. Instituto Humboldt, Bogotá. 2003, p. 261-76.

LATTKE, J. E. A taxonomic revision and phylogenetic analysis of the ant genus Gnamptogenys Roger in Southeast Asia and Australasia (Hymenoptera: Formicidae: Ponerinae). University of California Publications in Entomology, v. 122, p. 1-266, 2004.

LATTKE, J. E.; FERNANDÉZ, C. F.; PALACIO, E. E. Una nueva especie de Gnamptogenys (Hymenoptera: Formicidae) y comentarios sobre las especies del génereo en Colombia y Ecuador. Iheringia, Série Zoologia, v. 94, n. 4, p. 341-9, 2004.

LATTKE, J. E.; FERNÁNDEZ, F.; PALACIO, G. E. E. Identification of the species of Gnamptogenys Roger in the Americas. In: SNELLING, R.R.; FISHER, B.L.; WARD, P.S. (org). Advances in Ant Systematics (Hymenoptera: Formicidae): Homage to E. O. Wilson - 50 Years of Contributions. Memoirs of the American Entomological Institute, Gainesville, FL. 2007, p. 254-70.

LENOIR, J. C.; LACHAUD, J. P.; NETTEL, A.; FRESNEAU, D.; POTEAUX, C. The role of microgynes in the reproductive strategy of the neotropical ant Ectatomma ruidum. Naturwissenschaften, v. 98, p. 347-56, 2011.

LIMA, L.D; ANTONIALLI-JÚNIOR, W.F. Foraging strategies of the ant Ectatomma vizottoi (Hymenoptera, Formicidae). Revista Brasileira de Entomologia. Curitiba, v. 57, n. 4, p. 392-396, 2013.
MOREAU, C. S.; BELL, C. D.; VILA, R.; ARCHIBALD, S. B.; PIERCE, N. E. Phylogeny of the Ants:

Diversification in the Age of Angiosperms. Science. Washington, D.C, v. 312, p. 101-4, 2006.

OUELLETTE, G. D.; FISHER, B. L.; GIRMAN, D. J. Molecular systematics of basal subfamilies of ants using 28S rRNA (Hymenoptera: Formicidae). Molecular Phylogenetics and Evolution, v. 40, p. 35969, 2006.

PACHECO, J.; MACKAY, W.; MORGAN, C. A new species of Gnamptogenys Roger of the sulcata group (Hymenoptera: Formicidae) from Bolivia. Proceedings of the Entomological Society of Washington, v. 106, p. 434-7, 2004.

PACHECO, R.; VASCONCELOS, H. L.; GROC, S.; CAMACHO, G. P.; FRIZZO, T. L. M. The importance of remnants of natural vegetation for maintaining ant diversity in Brazilian agricultural landscapes. Biodiversity and Conservation, v. 22, p. 983-997, 2013.

PÉREZ-LACHAUD, G.; HERATY, J. M.; CARMICHAEL, A.; LACHAUD, J. P. Biology and behavior of Kapala (Hymenoptera: Eucharitidae) attacking Ectatomma, Gnamptogenys, and Pachycondyla (Formicidae: Ectatomminae and Ponerinae) in Chiapas, Mexico. Annals of the Entomological Society of America, v. 99, p. 567-76, 2006.

RABELING, C.; BROWN, J. M.; VERHAAGH, M. Newly discovered sister lineage sheds light on early ant evolution. Proceedings of the National Academy of Sciences of the United States of America, v. 105, p. 14913-7, 2008.

SHATTUCK, S. O. Australian Ants: Their Biology and Identification. CSIRO, Collingwood, Victoria, 2000.

TAYLOR, R. W. A checklist of the ants of Australia, New Caledonia and New Zealand (Hymenoptera: Formicidae). In: CSIRO Div. Entomol. Rep. CSIRO Division of Entomology Report, v. 41, p. 1-92, 1987.

VIEIRA, A. S.; ANTONIALLI-JUNIOR, W. F; FERNANDES W. D. Modelo arquitetônico de ninhos da formiga Ectatomma vizottoi Almeida (Hymenoptera, Formicidae). Revista Brasileira de Entomologia, v. 51, n. 4, p. 489-93, 2007.

VIEIRA, A. S.; FERNANDES, W. D.; ANTONIALLIJUNIOR, W. F. Temporal polyethism, life expectancy, and entropy of workers of the ant Ectatomma vizottoi Almeida, 1987 (Formicidae: Ectatomminae). Acta Ethologica, v. 13, p. 23-31, 2010. 
WARD, P. S. A systematic revision of the Rhytidoponera impressa group (Hymenoptera: Formicidae) in Australia and New Guinea. Australian Journal of Zoology, v. 28, p. 475-98, 1980.

WARD, P. S. A revision of the ant genus Rhytidoponera (Hymenoptera: Formicidae) in New Caledonia. Australian Journal of Zoology, v. 32, p. 131-75, 1984.

WU, J.; WANG, C. The ants of China. [in Chinese]. China Forestry Publishing House, Beijing. 1995.
XU, Z.; ZHANG, W. A new species of the genus Gnamptogenys (Hymenoptera: Formicidae: Ponerinae) from southwestern China. [in English, Chinese abstract]. Entomotaxonomia, v. 18, p. 55-8, 1996.

ZHOU, S. Ants of Guangxi. [In Chinese, English abstract and species descriptions]. Guangxi Normal University Press, Guilin. 2001. 